\title{
Nichtlineare Evolutionsgleichungen für $q$-holomorphe Vektoren ${ }^{\mathrm{i}}$ )
}

\author{
A. Crodel
}

Es werden Anfangswertprobleme für Evolutionsgleichungen in Räumen $q$ :holomorpher Vektoren betrachtet und für diese Sätze vom Cauchy-Kowalewskaja-Typ bewiesen.

Рассматриваются задачи Коши для әволюционных уравнений в пространствах $q$-голоморфиых векторов и доказываются -для них теоремы типа Коши-Ковалевской.

Initial value problems for cvolution processes are considered in spaces of $q$-holomorphic vectors and theorems of the Cauchy-Kovalevskaja type are proved for these problems.

In der Theorie partieller Differentialgleichungen stellt der Satz von Cauchy-Kowalewskaja eine zentrale Aussage dar. Eine Beweisvariante, die es uns erruöglicht, Lösungen durch sukzessive Approximation zu ermitteln, wird z. B. in [3] angegeben. . Verallgemeinerungen des klassischen Cauchy-Kowalewskaja-Satzes sind Grenzen gesetzt, wie das bekannte Lewysche Beispiel einer linearen Differentialgleichung ohne Lösung zeigt. Daß solche Vérallgemeinerungen jedoch prinzïpiell möglich sind, beweist eine Arbeit von W. TuTschke [6], in der ein Satz vom Cauchy-Kowalewskaja-Typ für verallgemeinerte analy tische Funktionen (im Sinne von I.N. Vekua) bewiesen wird. Die vorliegende Arbeit befaßt sich mit.Verallgemeinerungen des Satzes von Cauchy-Kowalewskaja für $q$-holomorphe Vektoren (im Sinne von B. W. BoJarski [1]). Wir betrachten Anfangswertprobleme der Form

$$
\frac{\partial w}{\partial t}=\dot{\mathscr{L}} w, \quad w(z, 0)=w_{0}(z)
$$

wobei $\mathscr{L}$ cin Operator ist, der Differentiationen nach der' räumlichen Variablen $z$ enthält. Es werden sowohl quasilineare als auch nichtlineare Gleichungen untersucht. Auf Besonderheiten des linearen Falles wird an entsprechender Stelle eingegangen. Der Beweis der Existenzsätze wird in einer Banachraumskala von $q$-holomorphen Vektoren geführt, wobei das abstrakte, nichtlineare Cauchy-KowalewskajaTheorem von T. NISHIDA [4] angewandt wird.

\section{Räume $q$-holọmorpher Véktoren}

Als $q$-holomorphe Vektoren bezeichnen wir die Lösungen des Systems

$$
l w:=\frac{\partial w}{\partial z^{*}}-q(z) \frac{\partial w}{\partial z}=0
$$

1) Vortrag auf der, Konferenz „Complex Analysis“ (8. Z. Anal. Anw. H. 2 (1986)). 
wobei $w=\left(w_{1}, \ldots, w_{n}\right)^{T}$ eine $\mathbf{C}^{\mathbf{n}}$-vektorwertige Funktion der komplexen Variablen $\boldsymbol{z}$ und $q$ eine Quasidiagonalmatrix

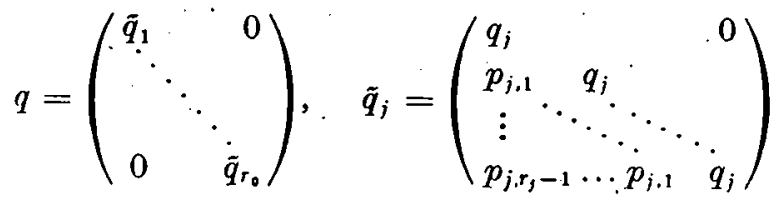

ist. Weiterhin soll $q=\left(q_{i, j}\right)$ die Abschätżung

$$
\sum_{i}\left|q_{i, j}\right| \leqq q_{0}<1 \quad \text { für alle } j \quad \text { ( } q_{0} \text { konstant) }
$$

erfüllen, die die Elliptizität des Systems (1.1) garantiert. Unter den Voraussetzungen $q \in W_{p}{ }^{1}(\mathbf{C}), p>2$, und $q \equiv 0$ außerhalb eines hinreichend großen Kreises wurde von B. W. BoJarSkI-[1] die Existenz einer ,erzeugenden Lösung " $\varphi \in C^{1, \alpha}(\mathbf{C})$ nachgewiesen. Diese ist eine Quasidiagonalmatrixfunktion; die in Konstruktion und grundlegenden Eigenschaften weitgehend dem Grundhomöomorphismus bei.Beltramigleichungen entspricht. Weiterhin wurde in [1] eine Cauchysche Integralformel für $q$-holomorphe Vektoren bewiesen. 'Später untersuchte B. GoLDschmIDT [2] funktionentheoretische Eigenschaften $q$-holomorphër Vektoren $w$, definierte die Ableitung Dw nach der erzeugenden Lösung $\varphi$ únd bewies die Bezichung $D w=\varphi_{z}^{-1} \partial w / \partial z$. Weiterhin gilt für stetige $q$-holomorphe Vektoren $w$ die Cauchysche Integraldarstellung der Ableitungen

$$
D^{k} w(z)=\frac{k !}{2 \pi i} \int_{\partial G} \varphi_{\zeta}(\zeta)[\varphi(\zeta)-\varphi(z)]^{-k-1} d_{q} \zeta w(\zeta)
$$

Zur Abkiurzung wurde dabei $d_{q} \zeta=I \cdot d \zeta+q \cdot d \zeta^{*}$ gesetzt.

Im weiteren werden wir voraussetzen, daß sowohl $q$ als auch $\varphi$ in einem Gebiet $\tilde{G} \subset \mathbf{C}$ bekannt sind. Es sei $G_{1}$ ein fest gewähltes Gebiet mit $G_{1} \Subset \widetilde{G}$. Durch Linearisierung von $\varphi$ erhält man mit einem gewissen $\delta_{0}$ die Abschätzung

$$
\left|[\varphi(\zeta)-\varphi(z)]^{-1}\right| \leqq \frac{r}{|\zeta-z|} \quad\left(\zeta, z \in \bar{G}_{1} \operatorname{mit} 0<|\zeta-z| \leqq \delta_{0}\right)
$$

Ist nun $G \subset G_{1}$, so kann man mit Hilfe dieser Ungleichung die Ableitung $D w$ von $q$-holomorphen Vektoren $w \in C(\bar{G})$ abschätzen. Es sei dazu $K \subset G$ ein Kompaktum, $\delta \leqq \min \left\{\delta_{0}, d(K, \partial G)\right]$ fest gewählt und $c=\sup \left\{\left|\varphi_{\zeta}(\zeta)\right|: \zeta \in \bar{G}_{1}\right\}$. Mit Hilfe der Cauchyschen Integralformel erhält màn dann für $z \in K$

$$
\begin{aligned}
|D w(z)| & \leqq \frac{1}{2 \pi} \int_{|\zeta-z|=\delta} \frac{\varphi_{\zeta}(\zeta)}{[\varphi(\zeta)-\varphi(z)]^{2}} d_{q} \zeta w(\zeta) \mid \\
& \leqq \frac{1}{2 \pi} \int_{\theta=0}^{2 \pi} \frac{c r^{2}}{|\zeta-z|^{2}}\left(1+\sqrt{n} q_{0}\right) d 0 \sup _{\bar{\sigma}}|w| \leqq \frac{c r^{2}\left(1+\sqrt{n} q_{0}\right)}{\delta} \sup _{\bar{\sigma}}|w|
\end{aligned}
$$

Diese Abschätzung gilt nur für $\delta \leqq \delta_{0}$. Bei der später verwendeten Methode zur Lösung von Anfangswertproblemen würde diese Einschränkung jedoch stören.' 
Setzen wir aber

$$
C=c r^{2}\left(1+\sqrt{n} q_{0}\right) \max \left(1, \operatorname{diam}\left(G_{1}\right) / 2 \delta_{0}\right),
$$

so erhalten wir das folgende

Lemma: Ist $w \in C(\bar{G})$ ein $q$-holomorpher Vektor und $K \subset G$ eine kompakte Teilmenge, so gilt mit $C$ uriabhängig von $G$.

$$
\sup _{K}|D w| \leqq \frac{C}{d(K, \partial G)} \sup _{\bar{G}}|w| .
$$

Beweis: Im Falle $2 \delta_{0} \geqq \operatorname{diam}\left(G_{1}\right)$ ist (1.3) identisch mit (1:2). Es sei nun : $2 \delta_{0}<\operatorname{diam}\left(G_{1}\right)$. Ist $d(K, \partial G) \leqq \delta_{0}$, so kann in (1.2) insbesondere $\delta=d(K, \partial G)$ gewählt werden, und (1.3) ergibt sich àus (1.2) durch Vergrößerung der Abschätzungskonstanten. Ist hingegen $d(\dot{K}, \partial G)>\delta_{0}$, so gilt wegen (1.2) mit $\delta=\delta_{0}$ ebenfalls

$$
\begin{aligned}
|D w(z)| & \leqq \frac{c r^{2}\left(1+\sqrt{n} \dot{q}_{0}\right)}{\delta_{0}} \sup _{\bar{G}}|w| \\
& \leqq \frac{c r^{2}\left(1+\sqrt{n} q_{0}\right)}{\delta_{0}} \frac{\operatorname{diam}\left(G_{1}\right)}{2 d\left(K, \partial G^{\prime}\right)} \sup _{\bar{G}}|w| \leqq \frac{C^{i}}{d(K, \partial G)} \sup _{\bar{G}}|w| .
\end{aligned}
$$

Die Unabhängigkeit von $C$ von der Wahl des Gebietes ist offensichtlich

Nun wollen, wir eine Skala von Banachräumen $q$-holomorpher Vektoren einfïhren. Dazu sei $\left(G_{s}\right)_{0<s \leq 1}$ eine Schar von Gebieten der komplexen Ebene, die für $s^{\prime}<s$ den Bedingungen

$$
\text { a) } \bar{G}_{s^{\prime}} \subset G_{s} \quad \text { und } \quad \text { b) } d\left(\bar{G}_{s^{\prime},}, \partial G_{s}^{\prime}\right) \geqq c_{d}\left(s-s^{\prime}\right) \text { mit } c_{d}>0
$$

gènügen soll.

Das Gebiet $G_{1}$ liege dabei kompakt in $\tilde{G}$. Ferner sei

$$
B_{s}=\left\{w \in C\left(\bar{G}_{s}\right) \cap W_{1,1 \mathrm{loc}}^{\mathrm{l}}\left(G_{s}\right) \mid / w=0\right\} \text { und }\|w\|_{s}=\sup _{z \in \bar{G}}|w(z)|
$$

Dann ist $\left(B_{s},\|\cdot\|_{s}\right)_{0 \leqq s \leqq 1}$ eine Skala von Banachräumen, die den Bedingungen $B_{s} \subset B_{s^{\prime}}$ uind. $\|\cdot\|_{s^{\prime}} \leqq\|\cdot\|_{s}$ für $s^{\prime}<s$ genügt. Betrachtet man nun den Ableitungsoperator $D$ in dieser Skala, so erhalt man mit $w \in B_{s}$ aus obigem Lemma die Abschätzung

$$
\|\dot{D} w\|_{s^{\prime}} \leqq \frac{C}{c_{d}\left(s-s^{\prime}\right)}\|w\|_{s} \quad \text { für } \quad s^{\prime}<s .
$$

Es gelingt also durch Einführung der Banachraumskala, die Unbeschränktheit des Differentialoperators $D$ zu umgehen, inden man $D$ als Operator auffaßt, der $B_{s}$ in $B_{s^{\prime}}\left(s^{\prime}<s\right)$ beschränkt abbildet. Dies gibt uns die Möglichkeit, Anfangswertprobleme der Form

$$
\frac{\partial w}{\partial t}=\mathscr{L} w, \quad w(z, 0)=w_{0}(z)
$$

wobei $\mathscr{L}$ den Operator $D$ enthält, durch sukzessive Approximation in der Banachraumskala $q$-holomorpher Vektoren zu lösen. 


\section{Quasilineare Evolutionsgleichungen}

Nun wollen wir Evolutionsgleichungen dèr Form

$$
\frac{\partial w}{\partial t}=\mathscr{L} w=A(w, z, t) \frac{\partial w}{\partial z}+F(w, z, t)
$$

untersuchen. Als Anfangsbedingung stellen wir

$$
w(z, 0)=w_{0}(z)
$$

mit einem $q$-holomorphen Vektor $w_{0} \in B_{1}$. Für die Lösbarkeit dieses Anfangswertproblems in der Skala $\left(B_{s}\right)$ spielt der folgende Assoziiertheitsbegriff cine wesentliche Rolle.

Definition: Der Differentialoperator $l$ heißt zum Operator $\mathscr{L}$ assoziiert, falls für jede Funktion $w$ mit $l w=0$ aus dem Definitionsbereich von $\mathscr{L}$ auch $l(\mathscr{L} w)=0$ gilt.

Es ist insofern zweckmäßig, eine solche Assoziiertheit vorauszusetzen, da für jede Lösung $w$ des Anfangswertproblems (2.1), (2.2) in der Skala $\left(B_{s}\right)$

$$
l(\mathscr{L} w)=l\left(\frac{\partial w}{\partial t}\right)=\frac{\partial}{\partial t}(l w)=0
$$

gilt, man jedoch die Lösung im allgemeinen nicht kennt. Bevor wir Bedingungen herleiten, unter denen die Operatoren $l$ und $\mathscr{L}$ assoziiert sind, sei noch ohne Beweis das folgende Lemma angegeben; das sich bei der Herleitung als nützlich erweisen wird.

Le $\mathrm{mm}$ m : Ist $M$ eine $n \times n$-Matrix und q.eine untere Drëiecksmatrix, deren Diagonalelemente sämtlich der. Bedingung $\left|\dot{q}_{i, i}^{\prime}\right|<1$ genügen, so folgt aus $M=q M q^{*}$ stets $M=0$.

Gehen wir von dem durch (2.1) definierten Differentialoperator $\mathscr{L}$ aus und wählen $w \in B_{s}$ aus der Definitionsmenge von $\mathscr{L}^{\prime}$, so ist

$$
\begin{aligned}
l(\mathscr{L} w)= & \left(\frac{\partial A}{\partial w} q-q \frac{\partial A}{\partial w}\right) \frac{\partial w}{\partial z} \frac{\partial w}{\partial z}+\left(\frac{\partial A}{\partial w^{*}}-q \frac{\partial A}{\partial w^{*}} q^{*}\right) \frac{\partial w^{*}}{\partial z^{*}} \frac{\partial w}{\partial z} \\
& +\left(\frac{\partial \dot{A}}{\partial z^{*}}-q \frac{\partial A}{\partial z}+\dot{A} \frac{\partial q}{\partial z}+\frac{\partial F}{\partial w} q-q \frac{\partial F}{\partial w}\right) \frac{\partial w}{\partial z} \\
& +\left(\frac{\partial F}{\partial w^{*}}-q \frac{\partial F}{\partial w} q^{*}\right) \frac{\partial w^{*}}{\partial \dot{z}^{*}}+(A q-q A) \frac{\partial^{2} w}{\partial z^{2}}+\left(\frac{\partial F}{\partial z^{*}}-q \frac{\partial F}{\partial z}\right)
\end{aligned}
$$

Dabei sind die' in den ersten beiden Summanden'auftretenden formalen Produkte in geeigneter Weise als Tensorprodukte aufzufassen. Da unabhängig von der Wahl von $w$ stets $l(\mathscr{L} w)=0$ gelten soll, ist es zweckmäßig, hier alle Koeffizienten Null zu setzen. Dies liefert unter Berücksichtigung des obigen Lemmas die folgenden' Assoziiertheitsbedingungen.

Satz: Es ist

$$
\iota w=\frac{\partial w}{\partial z^{*}}-\ddot{q} \frac{\partial w}{\partial z} \text { assoziiert } z u \mathscr{L} w=A(w, z, t) \frac{\partial w}{\partial z}+F(w, z, t),
$$


wenn die folgenden, Bedingungè a)-f) érfüllt sind:

a) $A$ und $F$ sind stetig bezüglich $z$ und besitzen lokal integrierbare Sobolevableitungen nach $z, z^{*}, w$ und $w^{*}$, -

b) $A q=q \dot{A}$,

c) $\frac{\partial A_{j}}{\partial w} q=q \frac{\partial A_{j}}{\partial w}$ für alle Spaltenvektoren $A_{j}$ von $A$,

d) $\frac{\partial F}{\partial z^{*}}-\because \frac{\partial F}{\partial z}=0$

e) $\frac{\partial A}{\partial z^{*}}-q \frac{\partial A}{\partial z}+A \frac{\partial q}{\partial z}=q \frac{\partial F}{\partial w}-\frac{\partial F}{\partial w} q$

f) $\frac{\partial A}{\partial w^{*}}=0$ und $\frac{\partial F}{\partial w^{*}}=0$.

Benterkung: Bedingung e) kann auch in der. Form

$$
\left(\frac{\partial}{\partial z^{*}}-q \frac{\partial}{\partial \dot{z}}\right)\left(A \varphi_{\dot{z}}\right)=\left(q \frac{\partial F}{\partial w}-\frac{\partial F}{\partial w} q\right) \varphi_{z}
$$

geschrieben werden, wobè $\varphi$ die erzeugende Lösung von $l w=0$ ist..

Um Existenzsätze für das Anfangswertproblem (2.1), (2.2) aufstellen zu können, benötigen wir nun noch, daß der Operator $\mathscr{L}$ die Skala $\left(B_{s}\right)$ lipschitzstetig in sich abbildet. Dies liefert der folgende

Satz: Es seien $A(w, z, t)$ und $F(w, z, t)$ für ein $\varepsilon>0$ stetig bezüglich aller ihrer Argumente in der Menge.

$$
\mathcal{M}_{R+\varepsilon}=\left\{(w, z, t)|| w-w_{0}(z) \mid<R+\varepsilon \text { für } z \in \bar{G}_{1} \text { und } \dot{t} \in[0, T]\right\}
$$

und die. Assoziiertheitsbedingungen (2.3) seien erfüllt. Dann bildet $\mathscr{L}$ die durch $\left\|w-w_{0}\right\|_{s} \leqq R$ definierte Kugel $K_{R}\left(w_{0}\right) \subset B_{s}$ in jedem Raum $B_{s^{\prime}}$ mit $s^{\prime}<s$ ab, und es gilt mit einer gewissen. Konstanten $\hat{C}$ die Ungleichung

$$
\left\|\mathscr{L} w_{1}-\mathscr{L} w_{2}\right\|_{s^{\prime}} \leqq \frac{\hat{C}}{s-s^{\prime}}\left\|w_{1}-w_{2}^{\prime}\right\|_{s} \text { für } w_{1}, w_{2} \in K_{R}\left(\dot{w}_{0}\right)
$$

Beweis: Aus der Stetigkeit und den Assoziiertheitsbedingungen folgt unter Berücksichtigung von $\partial w / \partial z=\varphi_{z} D w \in C\left(\bar{G}_{s^{\prime}}\right)$ zunächst $\mathscr{L} w \in B_{s^{\prime}}$. Da $A$ und $F$ weiterhin holomorph in $w$ sind, folgt aus der Stetigkeit in $\mathcal{M}_{R+\varepsilon}$ die Lipschitzstetigkeit bezüglich $w$ in $\overline{\mathscr{M}}_{R}$ (ánalog zu $\mathscr{M}_{R+\varepsilon}$ definiert). Die Lipschitzkonstanten seien dabei $L_{A}$ und $L_{F}$. Da die Menge $\vec{G}_{1} \times[0, T]$ kompakt ist; können diese unabhängig von $z$ und $t$ gewählt werden. Setzt man nun noch $M_{A}=\sup \left\{|A(w ; z, t)|:(w, z, t) \in \overline{\mathscr{M}}_{R}\right\}$, so erhält man unter Berücksichtigung von (1.4) durch elementare Abschätzungen

$$
\left\|\mathscr{L} \dot{w}_{1}-\mathscr{L} w_{2}\right\|_{s^{\prime}} \leqq \frac{\left[M_{A}+L_{A}\left(\left\|w_{0}\right\|_{1}+R\right)\right]\left\|\varphi_{z}\right\|_{1} C / c_{d}+L_{F}}{s-s^{\prime}}\left\|\dot{w}_{1}-w_{2}\right\|_{s}
$$

Weiterhin kann man noch abschätzen

$$
\left\|\mathscr{L} w_{0}\right\|_{s} \leqq \frac{\left\|A\left(w_{0}, \cdot, t\right)\right\|_{1}\left\|\varphi_{2}\right\|_{1}\left\|w_{0}\right\|_{1} C / c_{d}+\left\|F\left(w_{0}, \cdot, t\right)\right\|_{1}}{1-s}
$$


also

$$
\left\|\mathscr{L} w_{0}\right\|_{s} \leqq K /(1-s)
$$

wobei $K$ unabhängig von $t$ gewählt werden kann.

Bemerkung: Ist $\mathscr{L}$ ein linearer Operator der Form

$$
\mathscr{L} w=A(z, t) \frac{\partial w}{\partial z}+B(z, t) w+F(z, t)
$$

so erhält man für die Konstante $\hat{C}$ den Ausdruck

$$
\dot{\hat{C}}=\sup _{\ell}\|A\|_{1}\left\|\varphi_{z}\right\|_{1} C / c_{d}+\sup _{t}\|B\|_{1} \text {, }
$$

Nun wollen wir das Anfangswertproblem (2.1), (2.2) auf ein Problèn mit homogenen Anfangsdaten transformieren, indem wir als neue gesuchte Funktion $\tilde{w}(z, t)$ $=w(z, t)-w_{0}(z)$ einführen. Danit erhalten wir

$$
\begin{aligned}
& \frac{\partial \bar{w}}{\partial t}=\tilde{\mathscr{L}} \tilde{w}=A\left(\tilde{w}+w_{0}, z, t\right) \frac{\partial \bar{w}}{\partial z} \\
& \quad+A\left(\tilde{w}+w_{0}, z, t\right) \frac{\partial w_{0}}{\partial z}+F\left(\tilde{w}+w_{0}, z, t\right) \\
& \tilde{w}(z, 0)=0 .
\end{aligned}
$$

Faßt man dieses transformierte Problem als abstraktes Problem in der Skala von Banachräumen $\left(B_{s}\right)$ auf und berücksichtigt $w_{0} \in B_{1}$, so überzeugt man sich leicht, daß (2.6) die Voraussetzungen des abstrakten, nichtlinearen Cauchy-KowalewskajaTheorems von T. Nishida [4] erfüllt:

a) Der Operator $\tilde{\mathscr{L}}:\left\{\tilde{w} \in \dot{B}_{s} \mid\|\tilde{w}\|_{s} \leqq R\right\} \times[0, T] \rightarrow B_{s^{\prime}}$ ist für $s^{\prime}<s$ stetig.

b) Sind $\tilde{w}_{i} \in B_{s}$ mit $\left\|\tilde{w}_{i}\right\|_{s} \leqq R(i=1,2)$, so hat man wegen (2.4) für $s^{\prime}<s$ die, Abschätzung

$$
\left\|\tilde{\mathscr{L}} \bar{w}_{1}-\tilde{\mathscr{L}} \bar{w}_{2}\right\|_{s^{\prime}} \leqq \frac{c \hat{C}}{s-s^{\prime}}\left\|\bar{w}_{1}-\tilde{w}_{2}\right\|_{s} \quad \text { für alle } t \in[0, T] .
$$

c) Die Abbildung $\tilde{\mathscr{L}}(0, \cdot):[0, T] \rightarrow B_{s}$ ist stetig, und es gilt wegen (2.5)

$$
\|\tilde{\mathscr{L}}(0, t)\|_{s} \leqq K /(1-s) \text { für alle } t \in[0, T]
$$

Die Anwer̀dung des Nishidaschen Satzes liefert die Existenz und Eindeutigkeit einer Iuösung des Problems (2.6) in der Skala $\left(B_{s}\right)$. Dabei ist $\tilde{w}(\cdot, t) \in B_{s}$ für $t \in\left[0, T_{s}\right]$ mit

$$
T_{s}=\min [T, \dot{a}(1-s)] \quad \text { und } a=\min \left(\frac{2}{\dot{8} 1 \hat{C}}, \frac{3 R^{\prime}}{16 K\left(\pi^{2}-6\right)}\right) .
$$

Die Lösung $\tilde{w}$ ist beziiglich $t$ stetig differenzierbar und kann durch sukzessive Approximation berechnet werden.

Ist das Ausgangsproblem linear, so ist es günstiger, anstelle des Nishidaschen Satzes das lineare, abstrakte Cauchy-Kowalewskaja-Theorem (vgl. auch F. Treves [5]) anzuwenden: man erhält für ein beliebiges $\varkappa \in(0,1)$ die Lösung dann in einem größeren Zeitintervall mit $a=x /(e \hat{C})$. 
Für das Ausgangsproblem erhält man damit die folgende Aussage.

Satz: Erfüllen die Koeffizientenfunktionen $A$ und $F$ der Differentialgleichung

$$
\frac{\partial w}{\partial t}=A(w, z, t) \frac{\partial w}{\partial z}+F(w, z, t)
$$

die Assoziiertheitsbedingungen (2.3) und sind sie stetig in einer Umgebung einer Funktion $\dot{w}_{0} \in B_{1}$, so besitzt diese Gleichung unter der Anfangsibedingung $w(z, 0)=w_{0}(z)$ im Zeitintervall $\left[0, T_{s}\right]$, mit $T_{s} \stackrel{\min }{=}[T, a(1-s)]$ für eine gewisse Konstante a, eine im Gebiet $\vec{G}_{s}$ stetige und $q$-holomorphe Lösung $w$. Diese Lösung ist im Raum der q-holomorphen Vektoren eindeutig bestimmt.

Bemerkung: Die Lösung kann als Grenzwert der wie folgt definierten Folge konstruiert werden, wobei die Konvergenz für $(z, t) \in \bar{G}_{s} \times\left[0, T_{s}\right]$ gesichert ist:

$$
\begin{aligned}
& w_{1}(z, t)=w_{0}(z) \\
& w_{k+1}(z, t)=\dot{w}_{0}(z)+\int_{r=0}^{t}\left[A\left(w_{k}, z, \tau\right) \frac{\partial w_{k}}{\partial z}+F\left(\dot{w}_{k}, z, \tau\right)\right] d \tau .
\end{aligned}
$$

\section{Nichtlineare Evolutionsgleichungen}

Im folgendén sollen Anfangsẉertprobleme für nichtlineare Gleichungen der Form

$$
\frac{\partial w}{\partial t}=F(\dot{D} w, w, z, t)
$$

im Raum q-holomorpher Vektoren betrachtet werden. Eine direkte Behandlung dieses Problems mittels des Nishidaschen Satzes ist nur unter sehr einschneidenden Forderungen an $F$ möglich. Führt man jedoch $v=D w=\varphi_{z}^{-1} \partial w / \partial z$ als neue gesuchte Funktion ein, so kann man unter gewissen. Voraussetzungen Gleichung (3.1) auf ein quasilineares System zurückführen. Sind nämlich $\partial F / \partial w$ und $\partial F / \partial v$ mit $\varphi_{z}$ bezüglich der Matrizenmultiplikation vertauschbar und ist $F$ weiterhin holomorph in $v$ und $w$, so hat man

$$
\begin{aligned}
\frac{\partial v}{\partial t} & =\frac{\partial}{\partial t}\left(\varphi_{z}^{-1} \frac{\partial w}{\partial z}\right)=\varphi_{z}^{-1} \frac{\partial}{\partial z}\left(\frac{\partial w}{\partial t}\right)=\varphi_{z}^{-1} \frac{\partial}{\partial z}[F(v(z), w(z), z, t)] \\
& =\varphi_{z}^{-1}\left[\frac{\partial F}{\partial v} \frac{\partial v}{\partial z}+\frac{\partial F}{\partial w} \frac{\partial w}{\partial z}+\frac{\partial F}{\partial z}\right]=\frac{\partial F}{\partial v} D v+\frac{\partial F}{\partial w} D w+D F
\end{aligned}
$$

Mit den Bèzeichnungen

$$
\begin{aligned}
& \bar{w}=\left(\begin{array}{l}
w \\
v
\end{array}\right), \quad \tilde{F}=\left(\begin{array}{c}
F \\
D F
\end{array}\right), \quad \tilde{A}=\left(\begin{array}{cc}
0 & 0 \\
\partial F / \partial w & \partial F / \partial v
\end{array}\right) \\
& \dot{\bar{q}}=\left(\begin{array}{ll}
q & 0 \\
0 & q
\end{array}\right) \quad \text { und } \quad \tilde{\varphi}=\left(\begin{array}{ll}
\varphi & 0 \\
0 & \varphi
\end{array}\right)
\end{aligned}
$$

betrachten wir die Gleichung.

$$
\frac{\partial \tilde{w}}{\partial t}=\tilde{\mathscr{L}} \tilde{w}=\tilde{A}(\tilde{w}, z, t) \tilde{D} \tilde{w}+\tilde{F}(\bar{w}, z, t) \quad \text { mit } \quad \tilde{D} \tilde{w}=\tilde{\varphi}_{z}^{-1} \frac{\partial \dot{w}}{\partial z} .
$$


im Raunı der $\tilde{q}$-holomorphen Vektoren. Hierbei wird ausgenutzt, daß für $q$-holomorphe Vektoren $w$ auch die Ableitung $D w$ wieder $q$-holomorph ist. Elementare Rechnungen liefern den folgenden Satz, auf dessen Beweis wir hier nicht näher eingehen wollen.

Satz: Die Gleichung (3.1) ist unter der Anfangsbedingung .

$$
w(z, 0)=w_{0}(z)
$$

genau dann lösbar (bzw. eindeutig lösbar), wenn Gleichung (3.2) unter der Anfangsbedingung

$$
\tilde{w}(z, 0)=\left(\begin{array}{c}
w(z, 0) \\
v(z ; 0)
\end{array}\right)=\left(\begin{array}{c}
w_{0}(z) \\
\varphi_{z}^{-i} \frac{\partial}{\partial z} w_{0}(z)
\end{array}\right)=\bar{w}_{0}(z)
$$

lösbar (bzw. eindeutig lösbar) ist.

Auch in diesem Falle spielt wieder die Assoziiertheit der betrachteten Operatoren eine wesentliche Rolle. Unter entsprechenden Glattheitsforderungen an $F$ läßt sich dazu der folgende Satz beweisen.

Satz: Erfüllt $F$ die Bedingungen

a) $\frac{\partial F}{\partial w^{*}}=\frac{\partial F}{\partial v^{*}}=\dot{0}$
b) $\frac{\partial F}{\partial z^{*}}-q \frac{\partial F}{\partial z}=0$,

c) $\partial F^{\prime} / \partial w$ und $\partial F / \partial v$ sind bezüglich der Matrizenmultiplikation mit $q, \partial \dot{\partial} / \partial z$ und $\varphi_{z}$ vertauschbar,

- so sind folgende Operatorenpaare assoziiert:

$$
l w=\frac{\partial w}{\partial z^{*}}-\dot{q} \frac{\partial \dot{w}}{\partial z} z u \quad \dot{\mathscr{L}} w=F \cdot(D w, w, z, t)
$$

und

$$
\tilde{l} \tilde{w}=\frac{\partial \tilde{w}}{\partial z^{*}}-\tilde{q} \frac{\partial \tilde{w}}{\partial z} z u \quad \tilde{\mathscr{L}} \tilde{w}=\tilde{A}(\tilde{w}, z, t) \tilde{D} \tilde{w}+\tilde{F}(\tilde{w}, z, t)
$$

Béweis: Wir wollen diesen nur für $l$ und $\mathscr{L}$ führen (für $\tilde{l}$ und $\tilde{\mathscr{L}}$ verläuft er analog). Unter der Voraussetzung $l w=0$ ist

$$
\begin{aligned}
l(\mathscr{L} w)= & \left(\frac{\partial F}{\partial v} q-q \frac{\partial F}{\partial v}\right) \frac{\partial}{\partial z}(D w)+\left(\frac{\partial F}{\partial v^{*}}-q \frac{\partial F}{\partial v^{*}} q^{*}\right) \frac{\partial}{\partial z^{*}}(D w)^{*} \\
& +\left(\frac{\partial F}{\partial w} q-q \frac{\partial F}{\partial w}\right) \frac{\partial w}{\partial z}+\left(\frac{\partial F}{\partial w^{*}}-q \frac{\partial F}{\partial w^{*}} q^{*}\right) \frac{\partial w^{*}}{\partial z^{*}} \\
& +\left(\frac{\partial F}{\partial z^{*}}-q \frac{\partial F}{\partial z}\right)
\end{aligned}
$$

(dabei wurden schon die Beziehungen $\partial w / \partial \dot{z}^{*}=q \partial w / \partial z$ und $\partial(D w) / \partial z^{*} \stackrel{=}{=} q \partial(D w) / \partial z$ berücksichtigt). Da hier wegen (3.5) sämtliche Koeffizienten verschwinden, gilt stets $l(\mathscr{L} w)=.0$ 
Ist $\mathscr{L}$ als quasilinearer Operator gegeben und sind $l$ und $\mathscr{L}$ im Sinne der Bedingungen (2.3) assoziiert, so brauchen' die Bedingungen (3.5) im allgèmeinen nicht erfüllt zu sein.

Der folgende Satz sichert ab, daß der quasilineare Hilfsoperator $\tilde{\mathscr{L}}$ alle Voraussetzungen des Existenzsatzes aus Abschnitt 2 erfüllt.

Satz: Es sei.F auf der Menge $\overline{\mathscr{M}}_{R+e} \times[0, T]$ mit

$$
\mathscr{M}_{R+\varepsilon}=\left\{(v, w, z)|| v-\left.D \dot{w}_{0}(z)\right|^{2}+\left|w-w_{0}(z)\right|^{2}<(R+\varepsilon)^{2} \text { für } z \in \bar{G}_{1}\right\}
$$

definiert, wobei $w_{0} \in B_{1}$ die vorgegebene. Anfangsfunktion ist., Weiterhin sei $F \in C^{1}\left(\overline{\mathscr{M}}_{R+\varepsilon}\right) \cap W_{p}^{2}\left(\mathcal{M}_{R+\varepsilon}\right)$ für alle $t \in[0, T]$ und einem gewissen $p \geqq 1, F$ erfülle die Assoziiertheitsbedingungen (3.5) und sei stetig in $t$ bezüglich der $C^{1}\left(\overline{\mathscr{A}}_{R+\varepsilon}\right)$-Norm. Dann erfüllt der Operator $\widetilde{\mathscr{L}}$ alle Voraussetzungen des Existenzsatzes aus Abschritt 2. .

Beweis: Die Assoziiertheit von $\tilde{l}$ und $\tilde{\mathscr{L}}$ liefert der vorhergehende Satz, wobei die Existenz aller dort im Beweis auftretenden Ableitungen durch $F \in W_{p}^{2}\left(\mathcal{M}_{R+\ell}\right)$ abgesichert ist. Ferner, da $F \in C^{1}\left(\overline{\mathscr{A}}_{R+\ell}\right)$ stetig von $t$ abhängt, sind auch

$$
\tilde{A}=\left(\begin{array}{cc}
0 & 0 \\
\partial F / \partial w & \partial F / \partial v
\end{array}\right) \text { und } \tilde{F}=\left(\begin{array}{c}
F \\
D F
\end{array}\right)
$$

stetig in $\mathcal{M}_{R+c} \times[0, T]$, insbesondere also in einer Umgebung der Anfangsfunktion $\tilde{w}_{0}$

Wendét man nun den Existénzsatz aus Abschnitt 2 auf das quasilineare Problem (3.2), (3.4) in der Skala $\left(\tilde{B}_{s}\right)$ von $\bar{q}$-holomorphen Vektoren an, so erhält man aufgrüind dessen Aquivalenz zum Ausgangsproblem (3.1), (3.3) eine Lösung des letzteren; die zusammen mit ihrer Ableitung $D w$ in der Skala $\left(B_{8}\right)$ von $q$-holomorphen Vektoren liegt.

\section{Satz: Die Funktion $F$ in der Differentialgleichung}

$$
\frac{\partial w}{\partial t}+F(D w ; w, z, t)=\dot{F}(v, w, z, t)
$$

erfülle die Assoziiertheitsbedingungen (3.5) und sei nebst ihren Ableitungén nach $v, w$ und $z$ stetig in einer Umgebung. der Funktionen $w_{0} \in B_{1}:$ und $v_{0}=D w_{0} \in B_{1}$. Dann existiert eine Lösung $w$ mit $w(z, 0)=w_{0}(z)$, die nébst ihrer Ableitung Dw für $t \in\left[0, T_{s}\right]$ in $\bar{G}_{s}$ stetig ist. Dabei ist $T_{s}=\min [T, a(1-s)]$ und.a eine positive Konstante. Diese Lösung ist im Raum der $q$-holomorphen Vektoren eindeutig bestimmt.

Die Lösung unseres quasilinearen Hilfsproblems kann gemäß Formel (2.7) durch sukzessive Approximation konstruiert werden. Die $k$-te Iterierte $\bar{w}_{k}=\left(w_{k}{ }^{T}, v_{k}{ }^{T}\right)^{T}$ geniigt der Gleichung $D w_{k}=v_{k}$. Betrachten wir nämlich

$$
\begin{aligned}
\frac{\partial}{\partial t} D w_{k}(z, t) & =\frac{\partial}{\partial t}\left(\varphi_{z}^{-1} \frac{\partial w_{k}}{\partial z}\right)=\varphi_{z}^{-1} \frac{\partial}{\partial z}\left(\frac{\partial w_{k}}{\partial t}\right) \\
& =\varphi_{z}^{-1} \frac{\partial}{\partial z}\left[F\left(v_{k-1}(z), w_{k-1}(z), z, t\right)\right] \\
& =\frac{\partial F}{\partial v} D v_{k-1}+\frac{\partial F}{\partial w} D w_{k-1}+D F=\frac{\partial}{\partial t} v_{k}(z, t)
\end{aligned}
$$

und beachten, $\operatorname{da} B v_{k}(z, 0)=D w_{0}(z)=D w_{k}(z, 0)$. gilt, so erhalten wir gerade die oben behauptete Identität. Damit ist aber auch eine direkte sukzessive Approxi- mation der Lösung für das Ausgangsproblem möglich. Die Lösung des Problems 
(3.1), (3.3) kann also als Grenzwert der unitenstehend definierten Folge berechnet werden, wobei die Konvergenz fïr alle $(z, t) \in \bar{G}_{s} \times\left[0, T_{s}\right]$ gesichert ist:

$$
\begin{aligned}
& w_{1}(z, t)=w_{0}(z), \\
& w_{k+1}(z, t)=w_{0}(z)+\int_{r=0}^{t} F\left(D w_{k}, w_{k}, z, \tau\right) d \tau .
\end{aligned}
$$

Das quasilineare Hilfsproblem hat damit also lediglich technische Bedeutung für den Beweis der Existenz der. Lösung, zur Konstruktion wird es nicht benötigt.

Bei den hier durchgeführten Betrachtungen erscheint es als störend, da $B$ die $A b-$ leitung $D w$ als Argument der Funktion $F$ auftritt, da dies stets voraussetzt, da $B$ die erzeugende Iö̈sung $\varphi$ der Gleichung $l w=0$ bekannt ist. In gewissen Fällen ist es jedoch möglich, ohne die Kenntnis von $\varphi$ auszukomnien. Ist $F$ nämlich in der Form $F(\partial w / \partial z, w, t, z)=F(v, w, t, z)$ gegeben, so kann man stets wie folgt umformen:

$$
F(\partial w / \partial z, \dot{w}, z, t)=F\left(\varphi_{z} \dot{D} w, w, z, t\right)=\hat{F}(D w, w, z, t)=\hat{F}(\hat{\imath}, w, z, t) .
$$

Damit läßt sich das folgende Lemma formulieren.

Lemma: Erfüllt $F(\partial w / \partial z, \dot{w}, z, t)=F(v, w, z, t)$ die Bedingungen $(3.5)$, und ist $q$ in $G_{1}$ antiholomorph, so genügt auch $\hat{F}(D w, w, z, t)=\hat{F}(\hat{v}, w, z, t)$ diesen Bedingungen.

Beweis: Die Holomorphie von $\hat{F}$ in $\hat{v}$ und $w$ ist offensichtlich, ebenso Bedingung $(3.5) / c)$, wenn man beachtet, daß die Matrizen $q, \partial q / \partial z$ und $\partial \varphi / \partial z$ die gleiche Quasidiagonálstruktur besitzen und damit bezüglich der Matrizenmultiplikation untereinander vertauschbar sind. Weiterhin erhält man

$$
-{ }^{\prime}[\hat{F}(\hat{v}, w, z, t)]=l\left[F\left(\varphi_{z}(z) \hat{v}, w, z, t\right)\right]=\frac{\partial F}{\partial v} l\left(\varphi_{z}\right) v+\frac{\partial F}{\partial z^{*}}-q \frac{\partial F}{\partial z} .
$$

Da $q$ als antiholomorph vorausgesetzt war, gilt $l\left(\varphi_{z}\right)=0$. Aus der $q$-Holomorphie von $F$ folgt somit also auch Bedingung b)

Ist. $q$ also antiholomorph, so lassen -sich damit echt nichtlineare Probleme auch dann lösen, wenn die erzeugende Lösung $\varphi$ nicht explizit bekannt ist.

Die hier durchgeführten Untersuchungen lieferten Existenzsätze für Anfangswertprobleme nichtlinearer Evolutionsgleichungen. Hierbei wurden die Gleichungen stets in einem vorgegebenen Raum $q$-holomorpher Vektoren betrachtet. Interessant ist dieses Problem aber auch, wenn Lösungen in Räumen von verallgemeinerten analytischen Vektoren (im Sinne von B. W. Bojarski [1]) gesucht werden. Die assoziierte Differentialgleichung hat dann die
Gestalt

$$
w=\frac{\partial w}{\partial z^{*}}-q \frac{\partial w}{\partial z}-e w-j w^{*}=0
$$

In diesem Falle wird es jedoch nötig, anstelle der hier verwendeten Supremumsnorm mit der Höldernorm zu arbeiten, um die Beschränktheit (bzw. Lipschitźstetigkeit) des Operators $\mathscr{L}$ in der Skala zeigen zu können. Weiterhin ist es dann nicht mehr möglich, nichtlineare Fvolutionsgleichungen in einer ähnlichen Allgemeinheit zu behandeln wie im $q$-holomorphen Fall, da die in den Operatoren $l$ und $\mathscr{L}$ auftretenden Matrizen dann gewissen Nullteilerrelationen genügen müssen. Im Zusammenhang mit der Betrachtung solcher Probleme wird in einer späteren Arbeit auch auf die Frage eingegangen werden, wann zu ciner vorgegebenen Evolutionsgleichung eine assoziierte Differentialgleichung der obigen Form existiert. Insbesondere werden dann auch Bedingungen angegeben werden, unter denen die Koeffizienten $e$ und $f$ verschwinden und die Gleichung $l w=0$ somit einen Raum $q$-holomorpher Vektoren bestimmt. 
Die in der Arbeit bewiesenen Existenzsätze lieferten jeweils auch die Eindeutigkeit der . Lösung in der betrachteten Funktionenklasse, d. h. also im Raum der $q$-holomorphen Vekto- . ren. Offen ist hier noch die Frage nach der Eindeutigkeit der Lösung in umfassenderen Funktionenklassen.

\section{LITERATUR}

[1] Боярский, Б. В.: Теория обобщенного аналитического вектора. Ann. Polon. Math. 17 (1966), $281-320$.

[2] GoLdschmid, B.: Funktionentheoretische Eigenschaften verallgemeinerter analytischer , Vektoren. Math. Nachr. 90 (1979), $57-90$.

[3] Ниреньерг, Л:: Лекции по нелинейному фунициональному анализу: Москва: Изд-во Мир 1977.

[4] Nishid A, 'T.: A note on a theorem of Nirenberg. J. Diff. Geometry 12 (1977), 629-633.

[5] Treves, F.: Basic Linear Partial Differential Equations. New York: Academic Press 1975.

[6] Тучке, В.: Залача с начальными значениями для обобщенных аналитических функций, зависящих от времени. Докл. Акад. Наук СССР 262 (1982), 1081-1085.

[7] VEKUA, I: N.: Verallgemeinerte analy tische Funktionen. Berlin: Akademie.Verlag 1963.

Manuskripteingang: 14. 12. 1984

\section{VERFASSER :}

Andreas Crodel

Sektion Mathematik der Martin-Luther-Universität Halle - Wittenberg

DDR - 4010 Halle, Universitatsplatz 6 